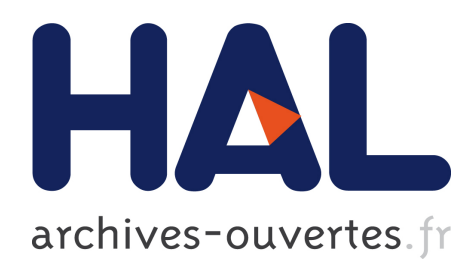

\title{
An automated assay for the assessment of cardiac arrest in fish embryo
}

\author{
Élodie Puybareau, Diane Genest, Emilie Barbeau, Marc Léonard, Hugues \\ Talbot
}

\section{To cite this version:}

Élodie Puybareau, Diane Genest, Emilie Barbeau, Marc Léonard, Hugues Talbot. An automated assay for the assessment of cardiac arrest in fish embryo. Computers in Biology and Medicine, Elsevier, 2016, <10.1016/j.compbiomed.2016.12.007>. <hal-01421156>

\section{HAL Id: hal-01421156 \\ https://hal.archives-ouvertes.fr/hal-01421156}

Submitted on 21 Dec 2016

HAL is a multi-disciplinary open access archive for the deposit and dissemination of scientific research documents, whether they are published or not. The documents may come from teaching and research institutions in France or abroad, or from public or private research centers.
L'archive ouverte pluridisciplinaire HAL, est destinée au dépôt et à la diffusion de documents scientifiques de niveau recherche, publiés ou non, émanant des établissements d'enseignement et de recherche français ou étrangers, des laboratoires publics ou privés. 


\section{(2)(1) $\$$}

Distributed under a Creative Commons Attribution - NonCommercial - ShareAlike 4.0 International License 


\title{
An automated assay for the assessment of cardiac arrest in fish embryo
}

\author{
Elodie Puybareau ${ }^{\mathrm{a}, *}$, Diane Genest $^{\mathrm{a}, \mathrm{b}, *}$, Emilie Barbeau $^{\mathrm{b}}$, Marc Léonard $^{\mathrm{b}}$, \\ Hugues Talbot ${ }^{a}$ \\ ${ }^{a}$ Université Paris-Est, LIGM (UMR 8049), CNRS, ENPC, ESIEE, UPEM, 2 Boulevard \\ Blaise Pascal, 93162 Noisy-le-Grand, France \\ ${ }^{b}$ L'OREAL Research 83 Innovation, Aulnay sous Bois, France
}

\begin{abstract}
Studies on fish embryo models are widely developed in research. They are used in several research field such as drug discovery or environmental toxicology. In this article, we propose an entirely automated assay to detect cardiac arrest in Medaka (Oryzias latipes) based on image analysis. We propose a multi-scale pipeline based on mathematical morphology. Starting from video sequences of entire wells in 24-well plates, we focus on the embryo, detect its heart, and ascertain whether or not the heart is beating based on intensity variation analysis. Our image analysis pipeline only uses commonly available operators. It has a low computational cost, allowing analysis at the same rate as acquisition. From an initial dataset of 3,192 videos, 660 were discarded as unusable (20.7\%), 655 of them correctly so $(99.25 \%)$ and only 5 incorrectly so $(0.75 \%)$. The 2,532 remaining videos were used for our test. On these, 45 errors were made, leading to a success rate of $98.23 \%$.
\end{abstract}

Keywords: Toxicology, medaka, segmentation, image stabilization, change detection, connected filtering

\footnotetext{
*These authors should be considered equal first authors

Email addresses: elodie.puybareau@esiee.fr (Elodie Puybareau), dgenest@rd.loreal.com (Diane Genest)

$U R L$ : www.ciliola.fr (Elodie Puybareau)
} 


\section{Introduction}

The use of fish embryo model as a potential alternative to laboratory mammalian species is increasing in a variety of physiological applications [1] [2]. In compliance with international animal welfare regulations, fish embryo mod5 els provide ethically acceptable systems having the complexity of a complete organism. More precisely, embryonic development in fish continues after hatching through the eleutheroembryo stage. At this stage, the energetic supply to the developing organism is provided by the yolk sac. The transition to the larval stage starts with the onset of exogenous feeding. [3, 4]. Eggs (before hatching) and eleutheroembryos do not meet the European regulatory definition of animals used for scientific purposes [5] and are therefore considered an alternative to (adult) animal testing [1, 6, 7]. However, evaluating effects of chemical compounds requires many measurements. Hundreds of measurements have to be conducted for each compound, with thousands of these chemicals being evaluated every year in the chemical, pharmaceutical, and cosmetic industries. These measurements had to be processed manually. Some can be done semi-automatically, but these approaches are not satisfying [8].

Image processing has been widely used in conjunction with fish studies, for instance for sizing and aging analysis [9, 10, species recognition [11, automated counting [12, behavior assessment [13, 14, and more recently for enabling micro-injections in fish embryo [15. Recent progress was achieved in automating image procedures analysis on Medaka embryos [16] and more commonly with Zebrafish embryos [17, 18, 19].

Fish embryos (Medaka and Zebrafish) are transparent and their cardiovascular system is readily visible. At these early stages of development, cardiac arrest does not induce an immediate death due to the blood gas exchanges that occur through skin diffusion [20, 21]. Nonetheless, we will refer to our cardiac arrest detection assay as a mortality test, since it can be considered as a prediction of mortality. Here we describe an automated image-processing

so pipeline to detect a beating heart with minimal human interaction, maximum 
speed, and reliability. The proposed procedure improves on a previous feasibility study [22], which had some limitations. In particular, it required a significant workload involving gel preparation and the manual positioning of embryos on the support gel. In addition, the number of plates used was limited due to a moving platform. In the procedure we will be describing in this article, freshly spawned eggs are placed in a liquid incubation medium, where they remain for 9 days, with automatic medium renewal every 3 days. This means having to conduct an image analysis of the eggs both unhatched and hatched in a liquid medium. This procedure involves using a fixed platform and a moving camera.

${ }_{40}$ The image analysis pipeline that we propose is based on mathematical morphology [23, 24, 25]. In the next section, we report the difficulties and challenges encountered in this study and their proposed solutions.

\section{Problem statement and challenges}

Our procedure is part of a complex process for detecting morphological and 45 functional abnormalities in fish embryos. This endeavor imposes some experimental constraints: we have to deal with both eggs and hatched eggs at the analysis level, and the embryos need to be anaesthetized during morphological analysis. Because this process is intended to be fully automated, a differentiating procedure between eggs and hatched eggs must be developed. We refer to the "hatched eggs" as alevins. We use the designation embryo to refer to eggs and alevins simultaneously. Medaka hearts normally beat at a frequency of around 130 beats per minute (bpm) i.e $2.2 \mathrm{~Hz}[26]$. However, this can vary by between 0 to $300 \mathrm{bpm}$, i.e $0-5 \mathrm{~Hz}$, in extreme cases. To avoid incorrect measurements, our recordings must be made using a frame rate that is high enough for our purposes. Our current camera records one second long videos at $30 \mathrm{~Hz}$, corresponding to a Nyquist cutoff frequency of $15 \mathrm{~Hz}$, which is sufficient. Recorded videos are $1500 \times 1500$ pixels in size, covering the whole well.

Because the incubation medium is liquid, undesirable motion may be present during acquisition. Embryos may also slide to the edge of each well, render- 
ing them partially or totally invisible. To minimize this, the platform is fixed and we use a moving camera. The quantity of liquid in the well is also carefully adjusted, so that the embryo moves as infrequently as possible once it is placed in the well. Incomplete or otherwise corrupted videos may occasionally be acquired. Shadows and undesirable objects are also a risk. These unusable 65 sequences must be identified at the start of processing.

Even with the above precautions and even if all of the embryos were to remain immobile under anesthesia, some residual movement is still possible. Such motion may be caused by involuntary reflex swimming, or it may be induced by vibrations and shocks in the lab environment, which are easily transmitted by the liquid in the well and on the embryo, inducing false positives if not corrected on dead embryos. In particular, the eyes can cause significant difficulties as they appear as the darkest part of the embryos' bodies and are not transparent . Consequently, we chose variance measures in the embryo's body excluding the eyes to detect heartbeats. However, artifactual motion caused by even the slightest uncompensated frame motion may induce artifacts of high variance and generate false positives. Because the heart is close to the eyes, this detection needs to be carefully handled. Moreover, in egg form, embryos are wounded and the eyes can superimpose with the heart, making the detection of a heartbeat impossible. More generally, eggs have different optical characteristics compared 80 to alevins. This is why it is important to determine whether a well contains an egg or an alevin so that the processing procedures can be adjusted accordingly.

During the nine-day incubation period in the chemical compound under study, the medium is regularly changed. At the nine-day mark, a fixed quantity of medium is removed from the well so that only $0.5 \mathrm{~mL}$ of liquid remains during image acquisition. The medium may still contain impurities or the chorion if the egg hatched during incubation. The real embryo must be carefully distinguished from these impurities during image processing. 


\section{Materials and Methods}

\subsection{Organism}

Medaka (Oryzias latipes) develops quickly (hatching normally occurs between 7 and 9 days post fertilization). In our study, embryos were procured from Amagen (UMS 3504 CNRS / UMS 1364 INRA). The eggs are incubated for 9 days at a temperature of $28^{\circ} \mathrm{C}$.

\subsection{Computer and software}

We used the Python 2.7 environment under Windows 7 (64 bits) in an HP computer with a $3.60 \mathrm{GHz}$ Intel $\AA$ Core ${ }^{\mathrm{TM}}$ i7-4790 CPU and $32 \mathrm{~GB}$ of RAM. We used Numpy, Scipy, and Scikit-image [27], Python Imaging Library (PIL), Pink [28], and Open CV for Python [29]. Platform control and image data acquisition were performed using FEI Visilog 7.

\subsection{Hardware}

During the incubation period, medium replacements were performed with an Hamilton MICROLAB STAR automated device. All videos were acquired using a monochrome camera (reference Basler Camera monochrome CCD 1" 2330*1750p 31 images/s Camera Link Mount C) and an acquisition platform from FEI.

\subsection{Acquisition protocol}

Fish eggs are manually placed in a 24 -well plate, one embryo per well, immediately after spawning on day 0 . Each well contains $2 \mathrm{~mL}$ of incubation medium, together with a predetermined concentration of the water-soluble compound under study. Embryos are incubated in this medium in their respective well until day 9, when image acquisition is performed. Medium replacements are automatically performed on days 2,5 and 7 . On day 9 , we remove $1.5 \mathrm{~mL}$ of the incubation medium from each well and the fish embryos are anesthetized with $70 \mu \mathrm{L}$ of tricaine $(1 \mathrm{~g} / \mathrm{L})$ in a total volume of $0.57 \mathrm{~mL}$. The final tricaine concentration is $0.1 \mathrm{~g} / \mathrm{L}$. The plate is placed under acquisition platform and recording 
is automatically performed under the control of a Visilog Visual Basic script.

For each well, 30 uncompressed video frames at a resolution of $1500 \times 1500$ pixels are recorded over a duration of $1 \mathrm{~s}$.

Due to storage constraints, sequence analysis cannot be performed offline. This means that it must take place in parallel to sequence acquisition. This imposes a maximum computation time of about 10 seconds. This limits the image analysis pipeline to simple, fast yet robust operators, preferably welltested and found in off-the-shelf image processing software packages.. On Fig. 1] we depict the flowchart of our assay. It consists of two steps: a stabilization and denoising phase, followed by a processing phase. The latter detects periodic changes in embryos associated with a beating heart. Videos are read as raw data interpreted as grey-level values.

\section{Image analysis solution}

In this section, we present our notations and image processing operators, mostly from mathematical morphology [31. Table 1 summarizes these. Let $I$ be a $M \times N$ pixels grey level image, taking 8 -bit discrete values, i.e. $I$ : $[1, N] \times[1, M] \rightarrow \mathbb{Z} \cap[0,255] . \quad B_{i}$ is a disk structuring element of radius $i$; $\varepsilon_{B_{i}}(I)$ is the erosion of $I$ by the structuring element $B_{i} . \delta_{B_{i}}(I)$ is the adjunct dilation [25], $\gamma_{B_{i}}(I)$ and $\varphi_{B_{i}}(I)$ are respectively the morphological opening and closing [30]. We denote $\gamma_{\lambda}^{\alpha}(X)$ the area opening of $X$ with parameter $\lambda$ [32]. We also denote $(I)_{\geq \theta}$ the binary, thresholded image of $I$ above value $\theta$, i.e.:

$$
x \in[1, N] \times[1, M],(I)_{\geq \theta}= \begin{cases}1 & \text { if } I(x) \geq \theta \\ 0 & \text { if } I(x)<\theta\end{cases}
$$




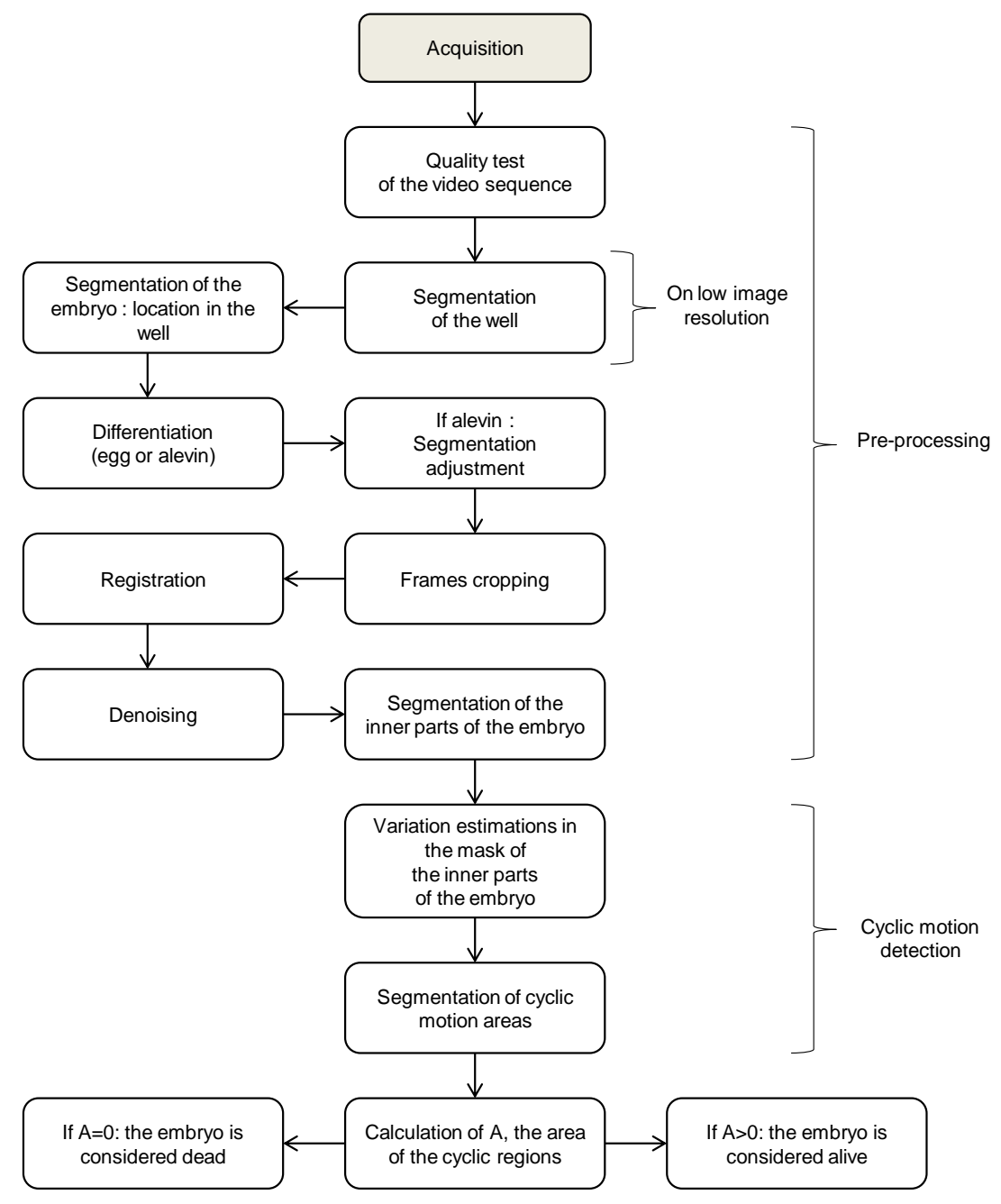

Figure 1: Flowchart of the embryo mortality image processing assay 
Table 1: Table of mathematical notations.

\begin{tabular}{|c|c|c|c|}
\hline Notation & Name & Definition & Reference \\
\hline$I$ & an $N \times M, 8$-bit image & $\forall p \in[1, N] \times[1, M], I(p) \in[0-255] \cap \mathbb{Z}$ & \\
\hline $\mathcal{S}^{l}$ & sequence $l$ of $n$ images & $\mathcal{S}^{l}=\left\{S_{i}^{l}, i \in\{1, \ldots n\}, S_{i}^{l}\right.$ an image $\}$ & \\
\hline $\mathcal{F}^{l}$ & first frame of the sequence $\mathcal{S}^{l}$ & $\mathcal{F}^{l}=S_{0}^{l}$ & \\
\hline V & pointwise maximum & & \\
\hline$\wedge$ & pointwise minimum & & \\
\hline$I_{v}$ & translation of image $I$ by vector $v$ & & \\
\hline$(I)_{\geq \theta}$ & Thresholding & $\forall p \in I, I(p)=1$ if $I(p) \geq \theta ; 0$ otherwise & \\
\hline$B_{i}$ & Euclidean disk of radius $i$ & & \\
\hline$\delta_{B}(I)$ & Dilation with structuring element $B$ & $\bigvee_{v \in B} I_{-v}$ & 30 \\
\hline$\varepsilon_{B}(I)$ & Erosion with structuring element $B$ & $\bigwedge_{v \in B} I_{v}$ & 30 \\
\hline$\varphi_{B}(I)$ & Closing with structuring element $B$ & $\varepsilon_{B}\left(\delta_{B}\right)(I)$ & 30 \\
\hline$\gamma_{B}(I)$ & Opening with structuring element $B$ & $\delta_{B}\left(\varepsilon_{B}\right)(I)$ & 30 \\
\hline bottom-hat $_{B}$ & Bottom-hat with structuring element $B$ & $\varphi_{B}(I)-I$ & 31 \\
\hline$\gamma_{\lambda}^{\alpha}(I)$ & Area opening & $\bigvee_{B, \text { area }(B)=\lambda} \gamma_{B}(I)$ & 32 \\
\hline$\rho_{\vartheta}^{\tau}$ & Segment of length $\tau$ and orientation $\vartheta$ & & \\
\hline$\gamma_{\tau}^{\rho}(I)$ & Radial opening & $\bigvee_{\vartheta \in[0, \pi]} \gamma_{\rho_{\vartheta}^{\tau}}(I)$ & 33 \\
\hline $\operatorname{grad}_{M}$ & Morphological gradient & $\delta_{B_{1}}(I)-\varepsilon_{B_{1}}(I)$ & \\
\hline$a=\operatorname{average}(I)$ & Scalar average & $a=\operatorname{average}(\{p, \forall p \in I\})$ & \\
\hline$\mu=\operatorname{median}(I)$ & Scalar median & $\mu=\operatorname{median}(\{p, \forall p \in I\})$ & \\
\hline$v=\operatorname{variance}(I)$ & Scalar variance & $v=\operatorname{variance}(\{p, \forall p \in I\})$ & \\
\hline$A=$ seq_average $(\mathcal{S})$ & Image average of sequence $\mathcal{S}$ & $\forall p, A(p)=\operatorname{average}\left(\left\{S_{i}(p), S_{i} \in \mathcal{S}\right\}\right)$ & \\
\hline$M=\operatorname{seq} \_\operatorname{median}(\mathcal{S})$ & Image median of sequence $\mathcal{S}$ & $\forall p, M(p)=\operatorname{median}\left(\left\{S_{i}(p), S_{i} \in \mathcal{S}\right\}\right)$ & \\
\hline$V=$ seq_variance $(\mathcal{S})$ & Image variance of sequence $\mathcal{S}$ & $\forall p, V(p)=\operatorname{variance}\left(\left\{S_{i}(p), S_{i} \in \mathcal{S}\right\}\right)$ & \\
\hline skeleton $(I)$ & Skeleton & & 34 \\
\hline watershed $(I)$ & Watershed & & 35 \\
\hline$G_{\sigma}(I)$ & Zero-mean Gaussian of variance $\sigma$ & $G_{\sigma}(p)=\frac{1}{\sigma \sqrt{2 \pi}} \exp -\frac{p^{2}}{2 \sigma^{2}}$ & \\
\hline $\operatorname{DoG}_{\sigma}(I)$ & Derivative of Gaussian at variance $\sigma$ & $\nabla \star G_{\sigma}(I)$ & 36] \\
\hline$\|\mathbf{v}\|$ & $\ell_{2}\left(\right.$ Euclidean) norm of vector $\mathbf{v}=\left(v_{x}, v_{y}\right)$ & $\sqrt{v_{x}^{2}+v_{y}^{2}}$ & \\
\hline
\end{tabular}


We now present the notion of radial opening [33]. Let $\rho_{\vartheta}^{\tau}$ be a line segment of length $\tau$ and orientation $\vartheta$. The radial opening $\gamma_{\tau}^{\rho}$ is the algebraic opening obtained by taking the supremum (i.e. the pointwise maximum) of all the openings $\gamma_{\rho_{\vartheta}^{\tau}}$ using $\rho_{\vartheta}^{\tau}$ as structuring element, with $\vartheta$ varying between 0 and $\pi$.

$$
\gamma_{\tau}^{\rho}(I)=\bigvee_{\vartheta \in[0, \pi]} \gamma_{\rho_{\vartheta}^{\tau}}(I)
$$

Intuitively, this opening preserves all structures in the image that can contain a segment of length $\tau$ in any direction. When $I$ is a binary image, the supremum operator $\bigvee$ reduces to the set union $\cup$.

We introduce the notation $\mathcal{S}^{l}$ to refer to a sequence $l$ of n images. We note $S_{i}^{l}$ the frame i of this sequence. For clarity reasons, we write $\mathcal{F}^{l}=S_{0}^{l}$ the first frame of the sequence $\mathcal{S}^{l}$.

\subsection{Video quality control and detection of unusable videos}

We begin by determining which videos present important and undesirable changes during the sequence. These changes may be due to the presence of black frames, shadows or large uncontrolled motion. For this, on each difference $d_{i}$ between two successive frames of the video sequence $\mathcal{S}^{0}$, we compute the statistical variance $\left(V_{i}\right)$ :

$$
\begin{gathered}
\forall i \in[1,29], d_{i}=S_{i}^{0}-S_{i+1}^{0} \\
V_{i}=\operatorname{variance}\left(d_{i}\right)
\end{gathered}
$$

In the case of a correctly recorded video, two successive frames should be very similar, and their pixelwise difference yields a near-zero output, so its variance remains small. On the contrary, if a large motion appears on a frame, the difference will show a high contrast. If at least one of all computed variances is higher than the experimentally determined threshold (set to 30), the video sequence is deemed unusable. 


\subsection{Segmentation of the well and selection of a region of interest}

Embryo segmentation is crucial for several reasons. For speed and reduced memory usage, we crop the area of interest to a small window centered on the embryo. During this step, we also detect sequences where the embryo is not fully visible, i.e. too close to the edges of the well. Moreover, motion stabilization must be performed on the embryo itself, and not on other elements of the field of view. We first isolate the region of interest by finding the disk area corresponding to the inner part of the well. This step also removes all objects connected to the edges of the well. The procedure for finding the area of this disk is as follows: edges of the disk appear dark, so we first compute a so-called bottom-hat filter: see Fig. 2(a) and Eq. (5) for a definition. In this equation, $B_{20}$ is chosen to remove small artifacts in the well. This yields image $A^{0}$ (Fig. 26) (b), which we binarize via an automated thresholding operation to obtain image $A^{1}$ in Fig. 2(c). The automated thresholding procedure is as follow: we determine the optimal threshold following the Otsu criterion, i.e. the one that maximizes the inter-class variance between two classes, foreground and background [37]), with the added constraint that the area of the foreground must be in the interval $[20,000 ; 40,000]$ pixels. This interval is experimentally determined to guarantee that the edges of the well are present in the foreground. This constraint is convex and so easily implemented: we consider all thresholds in order from the highest to the lowest in order. The foreground area necessarily increases during this process. In the acceptable foreground area interval for the area of the foreground, we select the threshold with the highest Otsu criterion. We call this threshold $\theta_{c o}$ (for constrained-Otsu).

$$
\begin{gathered}
A^{0}=\varphi_{B_{20}}\left(\mathcal{F}^{0}\right)-\mathcal{F}^{0} \\
A^{1}=\left(A^{0}\right)_{\geq \theta_{c o}}
\end{gathered}
$$

We remove small components from the well with an area opening $\gamma_{100}^{\alpha}$ of parameter $\lambda=100$, followed by a closing with a ball $B_{40}$ to reconstruct fragmented edges of the well. Then a radial opening $\gamma_{100}^{\rho}$ with linear element $\rho$ of length 


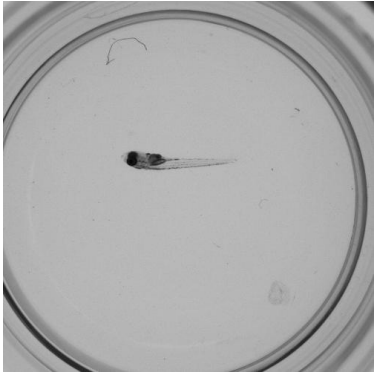

(a)

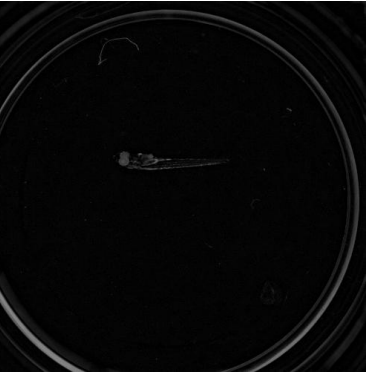

(b)

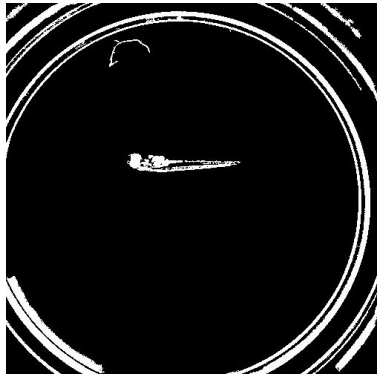

(c)

Figure 2: Bottom-hat application. (a) is the frame $\mathcal{F}^{0}$ before bottom-hat, (b) is the result $A^{0}$ and (c) is the subsequent thresholded image $A^{1}$.

$\tau=100$ is applied to remove short artifacts from the well, while retaining the thin well borders 33 . This yields image $A^{2}$ :

$$
A^{2}=\gamma_{100}^{\rho}\left(\varphi_{B_{40}}\left(\gamma_{100}^{\alpha}\left(A^{1}\right)\right)\right)
$$

From this result shown on Fig 3 (a), we only want to keep the internal ring that represents the separation between the interior of the well and its edges. For this, we use a well-established morphological approach to segmentation, based on the Watershed transform. This operator is intuitively defined as in hydrology, in a grayscale image whose intensity can be assimilated to a 3D terrain, as delimiting the borders between adjacent catchment basins [35]. We compute the magnitude of the Derivative of the Derivative of Gaussian filter (DoG): $\operatorname{DoG}_{\sigma}=\nabla \star G_{\sigma}$ using the Deriche recursive implementation of the gradient operator for speed [38] with parameter $\sigma=10$ and we use a markersbased Watershed algorithm [39] on the magnitude of this gradient. A disk at the center of the frame is taken as internal marker $m_{\mathrm{int}}^{1}$ and the frame corners are the external marker $m_{\text {ext }}^{1}$. We write:

$$
A^{3}=\operatorname{watershed}\left(\left\|\operatorname{DoG}_{10}\left(A^{2}\right)\right\|, m_{\text {int }}^{1}, m_{\text {ext }}^{1}\right)
$$

The resulting contour is shown on Fig 3 (b). The result may be incorrect if the embryo is too close to the edge of the well. To avoid this, we expand the 


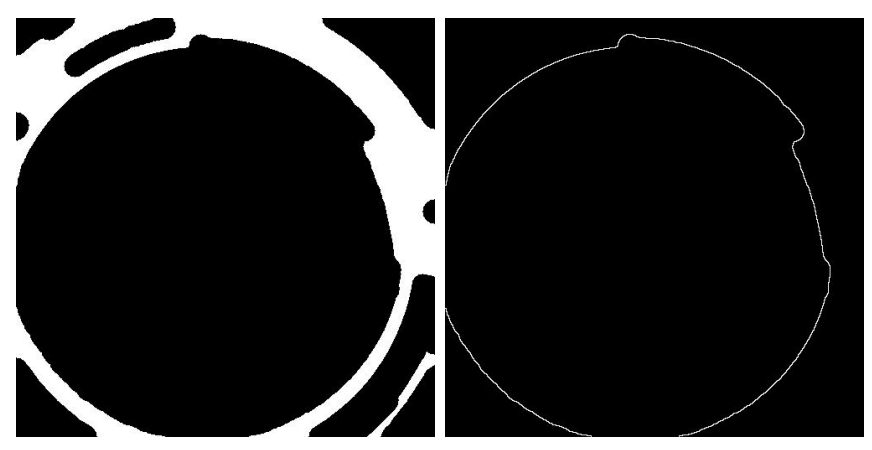

(a)

(b)

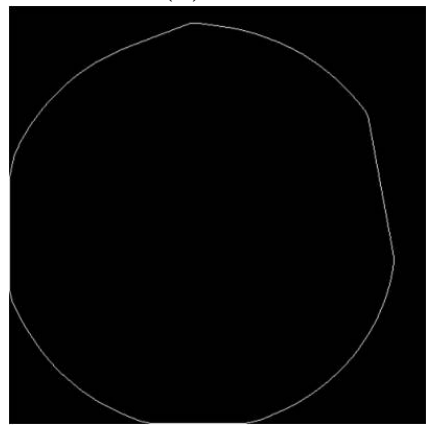

(c)

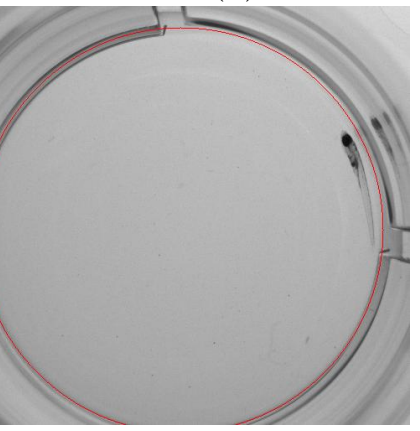

(d)

Figure 3: Segmentation of the inner part of the well. (a) is before watershed algorithm application (image $A^{2}$ ); (b) is the convex hull result (image $A^{3}$ ); (c) is the result of convex hull (image $A^{4}$ ) and (d) is the outline of final result $\mathcal{D}$ superimposed on $\mathcal{F}^{0}$. 
contour using the smallest convex set that contains $A^{3}$ [40]. We call $A^{4}$ the resulting image (see Fig. 3. (c)) and $G^{4}$ the set of points contained in the central component of $A^{4}$. We compute the barycenter $C$ of coordinates $(a, b)$ and the diameter $2 R$ of $G^{4}$ as the largest width or height of its bounding box. The final well segmentation is disk $\mathcal{D}$ centered in $C$ and of radius $R$. Its contour is shown on Fig 3 (d).

$$
\mathcal{D}=\left\{(x, y),(x-a)^{2}+(y-b)^{2} \leq R^{2}\right\}
$$

with $(a, b)=\operatorname{barycenter}\left(G^{4}\right)$ and $R=\frac{\max \left(\operatorname{width}\left(G^{4}\right), \operatorname{height}\left(G^{4}\right)\right)}{2}$

\subsection{Localization of the embryo in the well}

Our image analysis procedure is intended to work for both alevins and eggs, but some eggs do not develop at all and differ markedly from healthy eggs and alevins (see Fig. 4(a) and (c)). They feature low contrast, which makes them look like empty chorions or impurities that can develop in the wells. An early pipeline challenge is to reliably detect and identify the embryo in each well. To achieve this, we begin by performing an initial segmentation adapted to all components in the well, whatever their level of intensity or variance. In the previously calculated bottom-hat image $A^{0}$ (see Fig. 2(b)), all components of interest are easy to classify as connected components located strictly inside the segmented well. We call $h$ the contrast significance, understood as the intensity variation that connected components must have to be considered significant [24]. The value $h$ is experimentally set to ignore the irrelevant intensity variations due to noise, while still detecting the dimmest components that cannot be ignored (i.e. the undeveloped eggs). We call $p_{\text {peak }}^{i}$ the local maximum of intensity in the neighborhood of the pixel $p^{i}$. Image $B^{1}$ contains the so-called h-maxima of $A^{0} \cap \mathcal{D}$, defined as follows:

$$
B^{1}=\left\{p^{i} \in A^{0} \cap \mathcal{D} \text { with } p^{i}=\left[\begin{array}{ll}
0 & \text { if }\left(p_{\text {peak }}^{i}-p^{i}\right)>h \\
p^{i} & \text { if }\left(p_{\text {peak }}^{i}-p^{i}\right) \leq h
\end{array}\right\}\right.
$$

180

The image $B^{1}$ of the h-maxima can be efficiently computed using a morphological reconstruction operator, as explained in [41. 


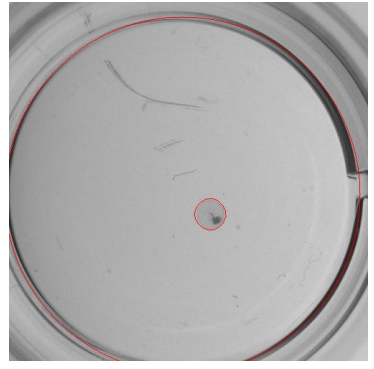

(a)

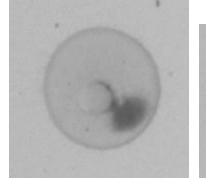

(c)

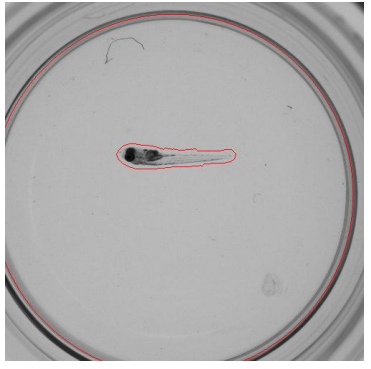

(b)

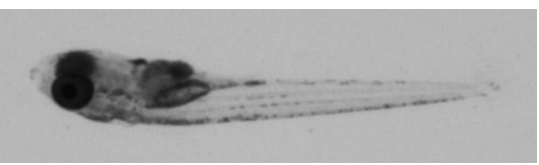

(d)

Figure 4: Segmentation of the well and location of the embryo. (a) and (b) show the outlines of $\mathcal{D}$ and $\mathcal{M}^{0}$ on the initial frame $\mathcal{F}^{0}$. (c) and (d) show the first frames of cropped sequences $\mathcal{S}^{1}$.

Several components can be detected in the resulting frame $B^{1}$. These components may be embryos, empty chorions, or some type of impurity. To identify the embryo, we use several criteria: presence of eyes, minimal and average intensities, variance, and circularity. A component is considered to have an eye if an extremely dark spot, representing less than a quarter of its total area, is present. Since impurities are generally homogeneous, this procedure filters out dark impurities, that are uniformly dark, and light impurities and chorions that are evenly bright. However, it can also filter out under-developed eggs. To avoid this problem, we add further classification criteria: a high average intensity or a low variance. We also verify circularity to differentiate between undeveloped eggs, chorions, and bright impurities. Finally, this process enables us to classify components as either "under-developed eggs," "other embryos," or "impurities and chorions." We delete components identified as impurities or chorions. There must only be one embryo per well. Therefore, if several components classified as "under-developped eggs" remain after this step, only the largest is kept. If sev- 
eral components of the other classes remain, we only keep the largest component among those from the "other embryo" class. Indeed, we have experimentally found that it is more difficult to distinguish under-developed eggs from chorions than other embryos from impurities. Thus the probability of making a mistake from the "undeveloped egg" class is higher. The result $\mathcal{M}^{0}$ is a binary mask (with values in $\{0,1\}$ ) containing only one component expected to locate the embryo in the well (see its red contour in Figure 4(a,b)). An empty result occurs when an embryo intersects the edges of the well. In this case, the corresponding sequence cannot be analyzed correctly. If an embryo is found, we crop the mask $\mathcal{M}^{0}$ and the sequence by defining a bounding box around the segmentation dilated by $B_{20}$, a disk of diameter 20 pixel. This results in a new sequence denoted $\mathcal{S}^{1}$ centered on the embryo (see Fig. [4(c)). However, because of contrast variations and the large variability of grey levels between embryos, the mask $\mathcal{M}^{0}$ is only approximate. In particular, for alevins, it delimits a rough area with the tail included (see Figure 4(b)) and potentially contains some shadows and impurities if they are too close to the embryo. For the purpose of heartbeat detection, we need to restrict the search field to the thorax region.

\subsection{Differentiation between eggs and hatched embryos}

Because they have different optical properties, it is necessary to identify the embryo type for further processing. The differentiation step is based on the morphological analysis of the embryo contours previously detected. The previous segmentation provides a reliable localization of the embryo, but only a rough approximation of its contours (see Fig 5 (a) and (b)), so these need to be refined. For this, we consider the first frame $\mathcal{F}^{1}$ of the cropped sequence $\mathcal{S}^{1}$. In particular, it is crucial to weed out potential shadows and impurities, which may have been segmented with the embryo, while retaining the tail segmentation for the alevins. We apply the same bottom-hat procedure as in Eq. (5) of section 4.2 to eliminate the background. Then we experimentally define an adaptive threshold slightly above the average pixel intensity near the border of the cropped frame. For our images, with a 8-bit depth, an increment of 5 was 
experimentally determined as appropriate: $\theta=\operatorname{average}\left(\mathcal{F}^{1}\right)+5$. We obtain a binary image, whose small components are filtered out with an area opening with parameter $\lambda=5$. We apply the morphological gradient of an image $I$ defined as $\operatorname{grad}_{M}(I) \equiv \delta_{B_{1}}(I)-\varepsilon_{B_{1}}(I)$ to the resulting image, to obtain image $C^{1}$.

$$
C^{1}=\operatorname{grad}_{M}\left(\gamma_{5}^{\alpha}\left(\left(\varphi_{B_{40}}\left(\mathcal{F}^{1}\right)-\mathcal{F}^{1}\right)_{\geq \theta}\right)\right)
$$

In order to properly extract the contours of the embryo without confusing them with residual artifacts that may still be present in the background, we again use a markers-based watershed methodology on the image $C^{1}$, as follows:

$$
\begin{aligned}
& m_{\text {int }}^{2}=\operatorname{skeleton}\left(\gamma_{B_{8}}\left(\varepsilon_{B_{15}}\left(\mathcal{M}^{0}\right)\right)\right) \\
& \mathcal{M}^{1}=\operatorname{watershed}\left(C^{1}, m_{\text {int }}^{2}, m_{\text {ext }}^{2}\right)
\end{aligned}
$$

The image's outline is set as the external marker $m_{\text {ext }}^{2}$ and the ultimate binary skeleton [42] of the eroded and opened mask $\mathcal{M}^{0}$ is set as the internal marker $m_{\text {int }}^{2}$. The erosion and the opening are respectively performed with a radius-15 and a radius- 8 disk, in order to remove potential thin impurities linked to the previous embryo segmentation.

The outline of the resulting binary mask $\mathcal{M}^{1}$ is shown in Fig. 5. (c) and (d).

We now use the shape of $\mathcal{M}^{1}$, to differentiate between the eggs and the alevins. Eggs are highly circular, so we can use the classical circularity attribute:

$$
\operatorname{circ}=\frac{4 \pi A}{P^{2}}
$$

Where $\mathrm{A}$ is the area and $\mathrm{P}$ is the perimeter of the binary shape under study. This ratio is at most equal to 1 for a disk, and decreases as the elongation becomes more pronounced. However, it is still possible for some alevins to be so tightly wound that their associated binary shape presents a high circularity. Eggs also possess hairs on their chorion that may reduce the circular aspect of their associated mask. To correctly differentiate between both cases, we also consider two other criteria. We have experimentally determined that a healthy 


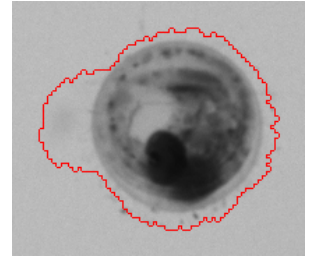

(a)

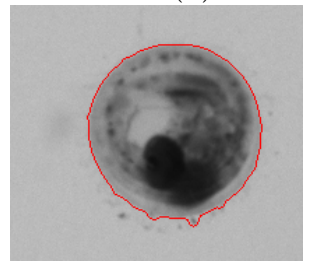

(c)

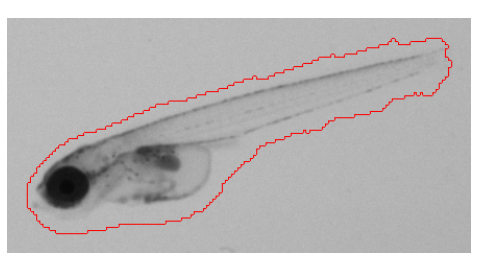

(b)

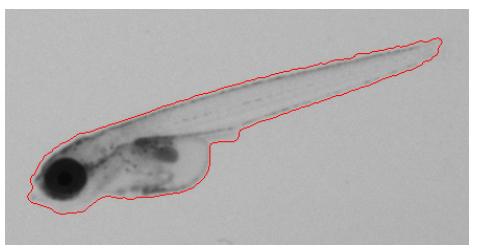

(d)

Figure 5: Segmentation of the embryo. $(\mathrm{a}, \mathrm{b})$ are the outline of the mask $\mathcal{M}^{0}$ (before adjustment) ; (c,d) are those of $\mathcal{M}^{1}$ (after adjustment).

well-segmented egg has a radius of around 60 pixels. Therefore, allowing for some margin of error, we apply a morphological opening $\gamma_{B_{40}}$ that deletes the alevins' mask, as alevins are much thinner than eggs. If the component under study is filtered out during this step, it is considered to be an alevin. If it is not, we determine the minimum enclosing disk of the mask and we calculate the area difference $d$ between this disk and the mask $\mathcal{M}^{1}$. Indeed, since the eggs' hairs are uniformly distributed on the chorion, the difference between the mask area and the area of its minimum enclosing disk is higher for hatched alevins than for eggs. Below an experimentally determined threshold of 3,000 pixels, we consider the component under study to be an egg. Otherwise, we conclude that it is an alevin.

\subsection{Segmentation of the thorax of hatched embryos}

For the purpose of heartbeat detection, it is essential to restrict the region of interest to the thorax, in order to minimize the probability of false detection due to electronic noise or blood flow in the bright tail regions. Therefore, after the differentiation step, we refine the embryo segmentation in the case of 


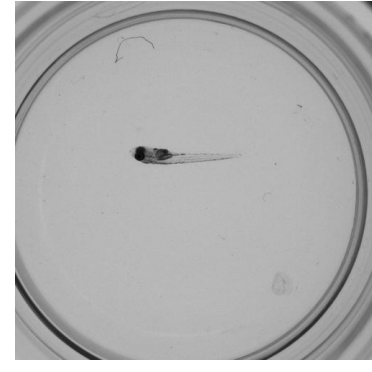

(a)

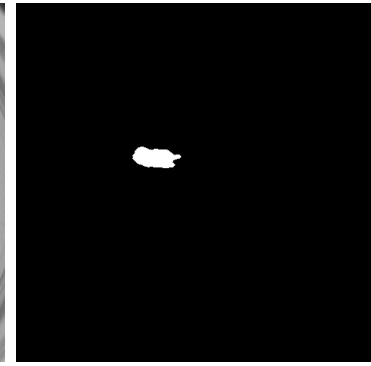

(b)

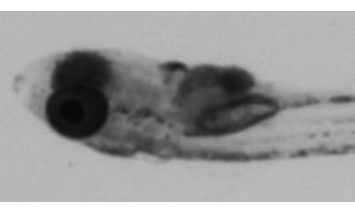

(c)

Figure 6: Segmentation of the initial frame to locate the thorax of the alevin. (a) is the initial frame $\mathcal{F}^{0}$, (b) the thorax mask $\mathcal{M}^{2}$ and (c) the first frame of $\mathcal{S}^{2}$

alevins. Alevins are darker than the background and their eyes, in particular, are very dark. They are fairly easy to segment as a large connected component associated with the darkest minima in the thorax region. We apply the same threshold process as in Eq. (6). Because we have experimentally determined that the minimal area of an alevin's thoracic region is approximately 600 square pixels, we apply a morphological area opening using the criterion $\lambda=100$ to eliminate small components. Moreover, we limit the thorax region to the $\mathcal{M}^{0}$, by computing the intersection.

$$
\mathcal{M}^{2}=\mathcal{M}^{0} \cap\left(\gamma_{100}^{\alpha}\left(\left(\mathcal{F}^{0}\right)_{\leq \theta_{o c}}\right)\right)
$$

The result $\mathcal{M}^{2}$ is a new binary mask representing the alevin's thorax (see Fig. 6(b)). We crop the sequence and obtain a new sequence $\mathcal{S}^{2}$ centered on this area (Fig. 6(c)). These remained unchanged for egg sequences and $\mathcal{M}^{2}$ and $\mathcal{S}^{2}$ are respectively equivalent to $\mathcal{M}^{1}$ and $\mathcal{S}^{1}$.

\subsection{Registration}

Even when anesthetized and subject to vibration isolation, embryos may still move slightly during acquisition. In order to eliminate false positives, all sequences need to be stabilized. For efficiency, we chose a keypoint based methodology, specifically using SIFT [43. SIFT detects and matches pairs of significant 
points $P_{1}$ and $P_{2}$ between pairs of frames. This allows us to solve the equation for rigid transformations:

$$
P_{1}=P_{2} \times R+T \text {. }
$$

Here $T=\left(d_{x}, d_{y}\right)$ is the translation vector and $R$ is the rotation matrix. Since embryos do not deform significantly, it is sufficient to consider this class of transforms. Our model can select between translation-rotations and translationonly transformations. Model selection is a useful feature, because simpler models are usually more robust. Here we distinguish between pure translation (where $R$ is the identity matrix) and translation-rotation by computing the sum of square difference between the two model outputs. If they do not differ significantly, we choose the simpler model. This latter outcome is the more frequent in our experiments. Pure rotation never occured in our experiments so we do not consider that model. Since impurities may be present in the well, movement is often visible around the embryo. Thus, in order to stabilize the sequence with respect to the embryo and not the other moving components in the well, we ensure that key-points in the embryo only are selected, restricting key-points to the mask $\mathcal{M}^{2}$. Taking the first frame of the sequence as reference, the selected model transform is applied to all the following frames. In order that the whole stabilized sequence be of constant width and height, we consider the bounding box of the sequence, and crop it by the maximum displacement in both $x$ and $y$, which are respectively $\max \left(\left|d_{x}\right|\right)$ and $\max \left(\left|d_{y}\right|\right)$. We call $\mathcal{S}^{3}$ the stabilized and cropped sequence of the embryo.

\subsection{Denoising}

Depending on the illumination conditions, sequence $\mathcal{S}^{3}$ may be more or less degraded by noise. We use a bilateral filter [44] in the $2 \mathrm{D}+\mathrm{t}$ domain to reduce noise. We can interpret the bilateral filter as a neighborhood-dependent convolution. At each pixel $(i, j)$ belonging to a window $W$, the filtered frame 
$\mathcal{I}_{D}$ is given by:

$$
\mathcal{I}_{D}(i, j, t)=\frac{1}{\sum_{(k, l, m) \in W} w(i, j, t, k, l, m)} \sum_{(k, l, m) \in W} I(k, l, m) \times w(i, j, t, k, l, m)
$$

Where

$$
w(i, j, t, k, l, m)=\exp \left(-\frac{\left((i-k)^{2}+(j-l)^{2}+(t-m)^{2}\right)}{2 \sigma_{d}^{2}}-\frac{\left.I(i, j, t)-I(k, l, m)^{2}\right)}{2 \sigma_{r}^{2}}\right)
$$

In this formulat, I is the original input image. Depending on parameters,

\subsection{Segmentation of the inner parts of the embryo}

To ascertain the presence of a heartbeat in the thorax region, we look for cyclic motion in this region only. To prevent false motion detection in unrelated areas, we compute a mask $\mathcal{M}^{3}$ corresponding to the region of interest. This eliminates areas most subject to noise, such that the eyes. We define $D^{1}$ as the sequential average image of the sequence $\mathcal{S}^{4}$ (see Table 1):

$$
D^{1}=\text { seq_average }\left(\mathcal{S}^{4}\right)
$$

Blood causes the heart and vessel to appear darker, so they are easy to segment as a large connected component associated with the darkest minima, simultaneously maximizing the inter-class variance.

$$
D^{2}=\left(D^{1}\right)_{\leq\left(\theta_{o}+\theta_{c}\right)}
$$

Here $\theta_{o}$ is obtained using the Otsu criterion. Because the heart and vessels are thin compared with the rest of the body, we need to bias the threshold 


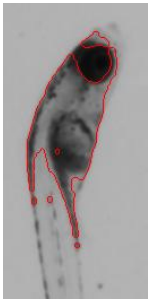

(a)

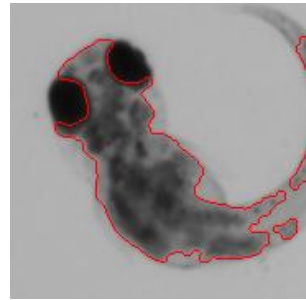

(b)

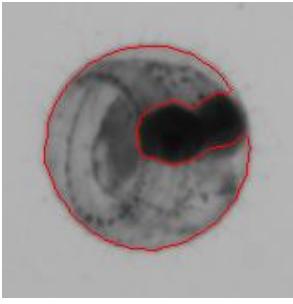

(c)

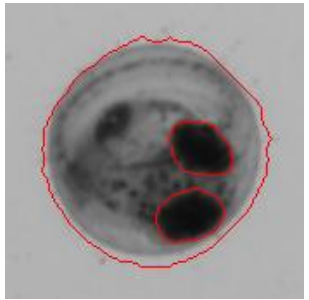

(d)

Figure 7: Segmentation of the inner parts of two alevins and two eggs. (a) and (c) are alive whereas (b) and (d) are dead.

to encompass a larger region, so we add a constant $\theta_{c}$ to the Ostu threshold, experimentally optimized to 20 . Here $D^{2}$ is a binary mask, corresponding to the body of the alevin, including the eyes. We detect the eyes in $D^{2}$ fairly simply, as they are large, obvious minima inside this region. We cannot assume that they eyes are separated but we do know their minimum size. The equation yielding $\mathcal{M}^{3}$ is:

$$
\mathcal{M}^{3}=\varepsilon_{B_{1}}\left(\gamma_{B_{3}}\left(\left(D^{2} \cdot D^{1}\right)_{\geq\left(\theta_{o}-\theta_{d}\right)}\right)\right)
$$

In this equation, $\theta_{o}$ is again the Otsu optimal threshold, which depends on the distribution of grey-levels within $D^{2}$. Because we want to bias the threshold nearer to the eyes, which are very dark,

we subtract an experimentally optimized constant $\theta_{d}$, which turns out to be equal to 20 as well, from $\theta_{o}$. The outline of the resulting mask $\mathcal{M}^{3}$ in alevins is exemplified in Fig. 7(a,b). This procedure is used only on alevins in order to restrict the region of interest to detect heartbeats. It is not suitable for eggs, due to the folded aspect of the embryo. For these, we compute $\mathcal{M}^{3}$ using the same procedure for segmenting the eyes but considering $\mathcal{M}^{2}$ as the geodesic mask. (Fig. 7( $(\mathrm{c}, \mathrm{d})$ ).

\subsection{Elimination of spurious, non-cyclic motion}

So far we have assumed that heartbeats can be associated with significant variations of grey-levels in the thorax region of the alevin. For this, we estimate 


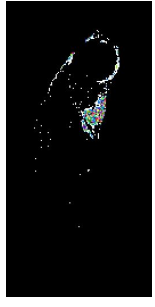

(a)

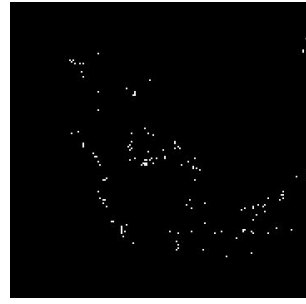

(b)

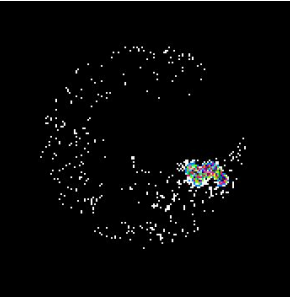

(c)

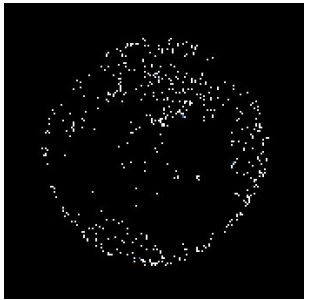

(d)

Figure 8: False color rending of the temporal variance. (a) and (c) are for the living embryos in Fig 7 (a,c); (b) and (d) for the dead ones in Fig 7 (b,d).

a time-wise, grey-level variance at every location in this region. However, a significant variance during a sequence may be also due to a single, large, spurious motion instead of a regular, periodic heartbeat. To distinguish between these two cases, we split $\mathcal{S}^{4}$ into four equal length sub-sequences. Each subsequence is 7 -frames long, which is enough to record a typical, single heartbeat. The sub-sequences are called $\mathcal{S}_{i}^{4}, i \in\{1 \ldots 4\}$. We now consider the sequential variance image $V_{i}=$ seq_variance $\left(\mathcal{S}_{i}^{4}\right), i \in\{1 \ldots 4\}$ and the sequential median $V=$ seq_variance $\left\{V_{i}\right\}$. We see if that a single, large, spurious motion occurs during the entire sequence, then only one of the $V_{i}$ will record high values. The median $V$ of the $V_{i}$ will still be low. In contrast, if a regular, signicant variations occurs in the majority of the $V_{i}$, then $V$ will have high values that we assume to be due to periodic motion. The result is shown on figure 8

$$
\begin{gathered}
\mathcal{S}^{4}=\mathcal{S}_{1}^{4} \cup \mathcal{S}_{2}^{4} \cup \mathcal{S}_{3}^{4} \cup \mathcal{S}_{4}^{4} \\
\forall i \in[1,4], V_{i}=\text { seq_variance }\left(\mathcal{S}_{i}^{4}\right) \\
E^{1}=\mathcal{M}^{3} \text {.seq_median }\left(\left\{V_{i}, i \in[1,4]\right\}\right)
\end{gathered}
$$

\subsection{Segmentation of cyclic motion areas}

We obtain a binary image of $E^{1}$ via a small closing, a threshold by the scalar median value $\mu$ of all strictly positive variances present in $E^{1}$, and a small area opening. 


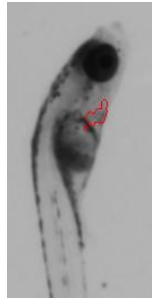

(a)

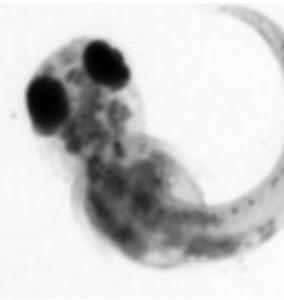

(b)

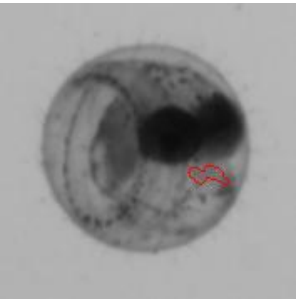

(c)

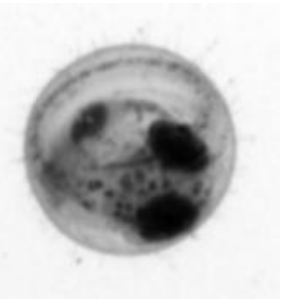

(d)

Figure 9: Segmentation of cyclic motion detection in embryos. (a) and (c) are for the living embryos in Fig 8(a,c); (b) and (d) are for the dead embryos in Fig 8, b,d).

$$
\begin{aligned}
\mu & =\operatorname{median}\left(E^{1}\right) \\
H^{1} & =\gamma_{4}^{\alpha}\left(\varphi_{B_{1}}\left(E^{1}\right)_{\geq \mu}\right)
\end{aligned}
$$

Because the optical properties of eggs are not the same as those of alevins, we notice more residual cyclic motion due to noise in the case of eggs. Therefore, we add an area opening with $\lambda=8$ only for eggs. If $H^{1}$ contains only zero-valued pixels, the embryo is considered dead. Otherwise it is assumed to be alive (see Fig. 9.

\subsection{Detection of cyclic motion associated with the mouth}

Some alevins have no detectable heartbeat in the sequence due to significant pigmentation in the thorax region. However, sometimes cyclic mouth motion induced by natural reflex demonstrates that the alevin is alive. To allow for these specific cases, we recommend the detection of cyclic mouth motion. Once again, we apply our cyclic motion detection and segmentation based on variance, but this time on the inverted mask $\mathcal{M}^{3}$. If an area of cyclic motion is detected $\left(\mathcal{M}_{\text {mouth }}\right)$, we estimate the distance between each component in this area and the alevin's eyes $\left(\mathcal{M}_{\text {eyes }}\right)$, by superposing both corresponding masks: $G^{1}=$ $\mathcal{M}_{\text {eyes }} \cup \mathcal{M}_{\text {mouth }}$, then performing a dilation of $G^{1}$ by a radius-14 ball: $G^{2}=$ $\delta_{B_{14}}\left(G^{1}\right)$. If a single area remains, the area of cyclic motion is close enough to 


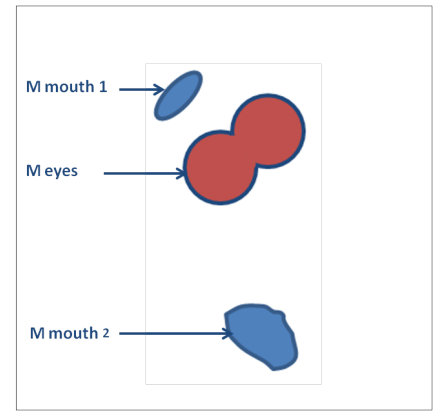

(a)

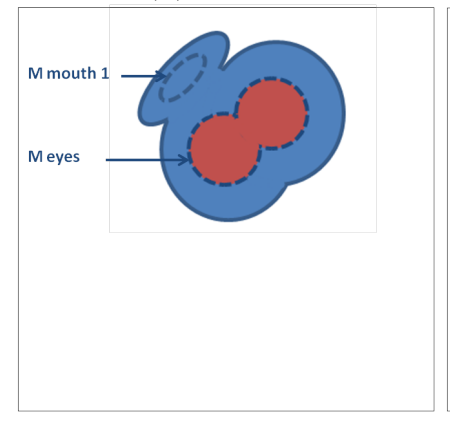

(c)

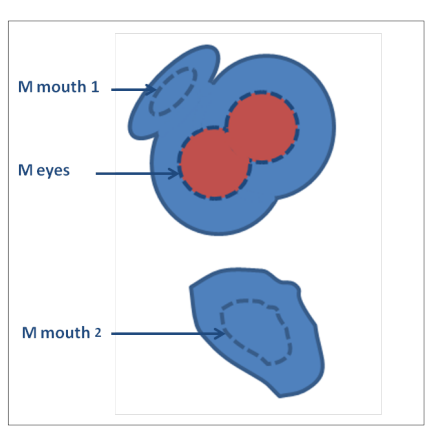

(b)

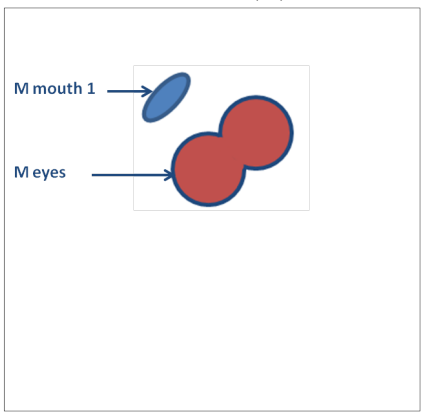

(d)

Figure 10: Distance assessment between areas of cyclic motion and alevin's eyes. (a) represents the superposed eyes and areas of cyclic motion masks $G^{1}$, (b) shows the dilation $G^{2}$, (c) is the component of $G^{3}$ which contains $\mathcal{M}_{\text {eyes }}$ and (d) is the result $G^{4}$.

the eyes to be the mouth. In terms of implementation, we keep the component of $G^{2}$ that contains the eyes, which we call $G^{3}$, and check to see if it also contains a component exhibiting cyclic motion (see Fig. 10.

$$
G^{4}=G^{3} \cdot G^{1}
$$

If the number of components in $G^{4}$ is higher than the number of eye components, we consider it a cyclic mouth motion area. An alevin with no heartbeat detected but mouth motion present is considered to be alive. 


\section{Validation and Discussion}

In this section, we present the results obtained using a total of 3,192 videos, 2,532 of which were actually usable to test for heartbeat detection. We begin describing the establishment of ground truth in part 5.1, before moving on to discuss processing. Our results are presented in part 5.3. We tackle the problem of remaining limitations in part 5.4 and finally discuss about the quality of our validation method in part 5.5

\subsection{Ground truth establishment}

In the context of our test validation, two possible types of ground truth exist: observations under a microscope and those directly acquired on videos. Each present different advantages and drawbacks. The strongest way of assessing the quality of the complete embryo analysis process, including plate preparation, data acquisition, and data treatment, is to compare our results to the observations of embryos under a microscope. On the other hand, the automated method we developed works on video sequences whose information may be much different from the observations made under microscope. Several aspects linked to the experimental protocol or the acquisition method can explain this fact. (i) Video quality is such that some weak heart beats may be undetectable on video even if they are visible under a microscope. (ii) Observations made while using a microscope also depend on operator fatigue and subjectivity. (iii) Because there is a time gap between the observations made under microscope and videos acquisition, an embryo may also die during the interval. (iv) Observations made under microscope facilitate scrutiny of the heart since embryos can be moved to a favorable position, whereas in videos the embryo's posture may obscure the heart. For these reasons, it appears that the most relevant way to assess the program's quality (one of the goals of this article) is through direct video observation.

In the context of our study, both possible ground truths were considered. In the table 2 summarizes the establishment of ground truth datasets. Our first 


\begin{tabular}{|c|c|c|c|}
\hline & Dataset 0 & Dataset 1 & Dataset 2 \\
\hline Screening method & Microscope & Videos & Videos \\
\hline Dataset size & 3192 & 3192 & 200 \\
\hline $\begin{array}{l}\text { Experts in charge } \\
\text { of the analysis* }\end{array}$ & Expert 0 & Expert 1 & $\begin{array}{c}\text { Experts } 1,2 \\
\text { and } 3\end{array}$ \\
\hline $\begin{array}{l}\text { Classification } \\
\text { labels used }\end{array}$ & "Alive" or "Dead" & $\begin{array}{c}\text { "Unusable", } \\
\text { "Alive" or "Dead" }\end{array}$ & "Alive" or "Dead" \\
\hline
\end{tabular}

* Each expert screened the entire dataset.

Table 2: Establishment of ground truth datasets. For Dataset 2, a consensus was reached between the three experts and a final set of 200 ground truth data was obtained.

expert (named "expert 0") originally observed the embryos under microscope before the total set of 3,192 videos were acquired. We refer to these observations as "Dataset 0". The expert identified each case as a living or dead embryo by checking for the presence of a heartbeat. After that, another expert ("expert 1 ") analyzed the resulting 3,192 acquired videos ("Dataset 1"). He began manually assessing the usability of the videos, by checking that they were complete, wellrecorded, that the well was not empty, and that the embryo was not too close to the well boundary. In this manner, 655 unusable videos were identified. Then, for the remaining 2,537 usable sequences only, this expert determined if the embryo was alive or dead. However, whereas determining videos usability is easy and thus reliable, detecting a beating heart is sometimes difficult and therefore subject to errors. For this reason, we selected a subset of only 200 usable videos ("Dataset 2") so that the health status of the embryo could be reassessed by two other independent observers ("experts 2 and 3"). In the end, three different observers were involved in analyzing the 200 usable data subset. 

identical, a consensus was then reached between these three observers concerning the videos that they assessed differently. The 200 data resulting from this consensus represent the ground truth we use to validate our automated method, as explained in the following section 5.3

\subsection{Processing}

Our heartbeat detection method returns three possible results: "unusable," "alive," or "dead." This method processes a sequence in approximately $10 \mathrm{sec}-$ onds, in accordance with our initial constraints. All parameters were hand optimized using a training sample of 100 sequences.

\subsection{Validation of the method}

Using the 200 Dataset 2 videos, we can now compare the results of our program with the previously established consensus data. We consider the program to be erroneous if it detects a dead embryo that was identified as alive according to expert consensus (false negative) or if, on the other hand, it detects a living embryo that was identified as dead by expert consensus (false positive). We detected 3 errors made by the program for a corresponding error rate of $1.5 \%$. (see Table 3). Moreover, this error rate only corresponds to false negatives. In toxicity tests, this is a more acceptable type of error since it does not provide a false sense of security with regard to the molecule tested. In Table 3(b), we present the error rates that were calculated for each expert as compared to the final consensus based on Dataset 2. Expert 1, who processed the 3,192 videos (Dataset 1), has a similarity rate of $98.5 \%$ with respect to the consensus data. Consequently, the experts' observations can be considered sufficiently reliable to analyze the results of the entire program. The results of this analysis (program vs. expert 1) are described below.

Out of the initial 3,192 Dataset 1 videos, the program correctly flagged 655 as unusable and incorrectly flagged another 5 as unusable due to some error within the program itself. If we consider the entire set, 3,187 videos were correctly 


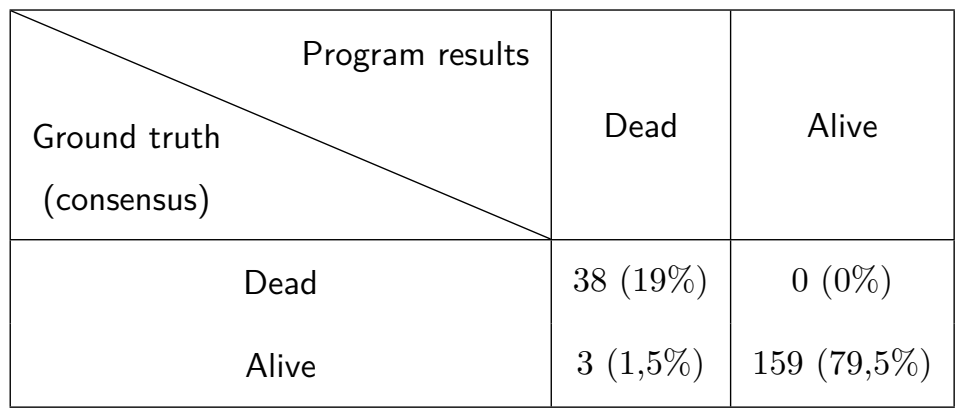

(a)

\begin{tabular}{|c|c|c|c|c|}
\hline & Expert 1 & Expert 2 & Expert 3 & Program \\
\hline $\begin{array}{c}\text { Error rates between } \\
\text { expert observations } \\
\text { and ground truth (consensus) }\end{array}$ & $1.5 \%$ & $2.5 \%$ & $1.5 \%$ & $1.5 \%$ \\
\hline
\end{tabular}

(b)

Table 3: Results and error rates calculated on Dataset 2 (200 usable videos). (a) shows the distribution of dead and living embryos in the program results compared to ground truth data of the consensus. It shows that $1,5 \%$ of the Dataset 2 embryos are wrongly identified as dead by the program. (b) shows the error rates calculated for each expert and for the program versus consensus data, used as ground truth.

flagged, leading to a success rate of $99.85 \%$. The remaining 2,532 videos were used for mortality test validation. There were 45 errors in this set, for an error rate of $1.77 \%$. Such an error rate is low and can be considered satisfactory. We noticed that 11 of these 45 errors were due to embryos that had died a long time before acquisition and had consequently absorbed the blue marker. These embryos appear very dark on the video and are therefore more affected by noise, which was incorrectly labeled as periodic motion. This is something we can improve in a future version of our software pipeline. 


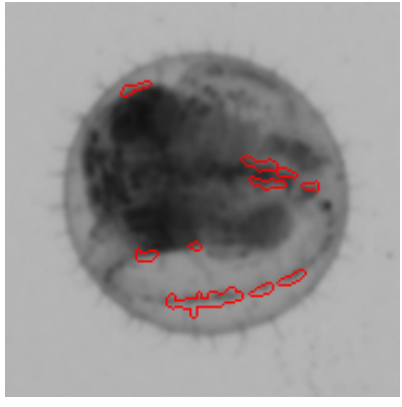

(a)

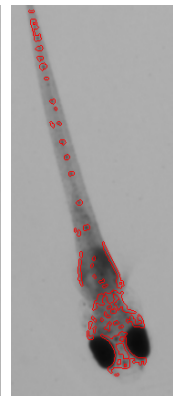

(b)

Figure 11: Incorrect segmentations due to fluttering.

\subsection{Limitations and further optimization}

In some cases, even embryos that are dead may appear to move. This may be caused by movement in the water, fluttering, shadows or embryo rotation inside the well (see Fig. 11 for an illustration). In dark areas, acquisition noise is proportionnally more troublesome [45], and may be confused with cyclic motion. Artefact motion may be present if we do not correctly stabilize the initial sequence. This may happen if motion in the original sequence is too large or too rapid or both, which is infrequent. Finally, the heart may beat too slowly or weakly. In these cases, it would be difficult for a human to detect it also.

Conversely, because embryo health status is assessed under the microscope before videos acquisition, an embryo may be identified as alive during microscopic analysis and die during the time gap. To avoid this, we plan to assess the embryo twice in our future studies: both before and after video analysis so that heart failure can be detected.

\subsection{Qualitative assessment of the validation method}

We tested our procedure on a total set of 3,192 videos acquired over several experimental runs. This is a significant number of videos containing healthy as well as diseased embryos: some with edemas and other malformations. Thus, this set reflects production usage and allows us to validate the robustness of our protocol (see Fig. 12. 


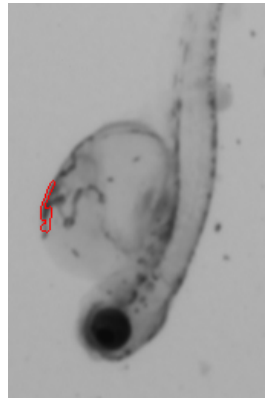

(a)

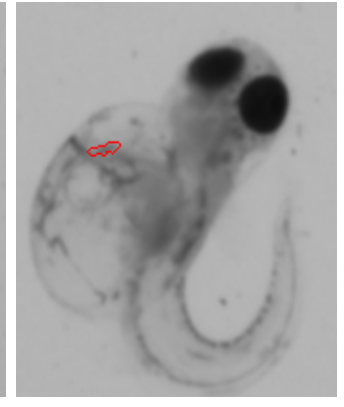

(b)

Figure 12: Heart segmentation in the presence of malformations. (a) is in the presence of edema and (b) in the presence of axial malformation.

The purpose of this article is to present and assess an image analysis pipeline for detecting a beating heart on 1 second long videos of fish embryos. Our results on 2,532 usable sequences show an acceptably low error rate, near $1.5 \%$ overall. This proves the efficacy and reliability of our image analysis method. However, when considering its integration within the entire system of embryos preparation, image acquisition, and processing, several points remain to be discussed, especially with respect to the validation phase and the establishment of ground truth.

Since we are discussing living organisms, establishing ground truth is not always easy. We rely on multiple visual observations of a subset of video sequences, which were not always consistent: expert observers did not always come to same conclusion. Indeed, we noticed 6 differences between them for 200 assessed videos, a rate of $3 \%$. A second viewing of these videos was consequently performed with all observers present to achieve a consensus. With respect to this consensus, each observer had made between 3 and 5 errors, a rate between $1.5 \%$ and $2.5 \%$. We note that our program had also made 3 errors. We conclude that the rate of assessment subjectivity is near 1.5\%. Such a rate is acceptable, because experiments may easily be repeated three times.

Assuming errors are random and independent, the final error rate on repeated 
experiments is negligible $\left(2.25 \times 10^{-4}\right)$.

However, this low error rate is based on video-based ground truth and is only representative of the quality of the program itself. To assess the reliability of the entire procedure, including preparation, acquisition, and treatment, we

\section{Conclusion}

In this article, we have detailed an image analysis pipeline for detecting the living status of a Medaka embryo. This is achieved by locating the area around 

which are assumed to correspond to a heartbeat. We have optimized the parameters of the proposed procedure on a small subset of 100 sequences. A large set of 2,537 videos was used as a test set, with these parameters fixed. By comparison to multiple human observers, we have round that the error rate of the video analysis is only $1.77 \%$, which is similar to the error rate human observers have between themselves. This error rate is sufficiently low for production use. Plans for future work include improvement of the experimental protocol so that the optimal viewing position for automated image analysis is attained and experimental errors are avoided. Our proposed pipeline is in production usage at

\section{Acknowledgment}

We thank Noémie De Croze and Mylene Rialland from the L'Oréal environmental research team in Aulnay sous Bois for their expertise, the material provided, and their essential contribution during this project.

\section{References}

[1] M. R. Embry, S. E. Belanger, T. A. Braunbeck, M. Galay-Burgos, M. Halder, D. E. Hinton, M. A. Léonard, A. Lillicrap, T. Norberg-King, G. Whale, The fish embryo toxicity test as an animal alternative method in hazard and risk assessment and scientific research, Aquatic Toxicology (97) (2010) 79-87.

[2] R. Nagel, Dart - the embryotest with the zebrafish danio rerio - a general model in ecotoxicology and toxicology, Alternativen zu Tierexperimenten (19) (2002) 38-48.

[3] E. K. Balon, Types of feeding in the ontogeny of fishes and the life history model, Environmental Biology of Fishes. 
[4] S. E. Belanger, E. K. Balon, J. M. Rawlings, Natural thresholds in the early ontogeny of fishes related to definitions of protected and non-protected life intervals, Aquatic Toxicology.

[5] Directive 2010/63/eu of the european parliament and of the council of 22 september 2010 on the protection of animals used for scientific purposes, Official J Eur Union 276.

[6] M. Halder, M. Léonard, T. Iguchi, J. T. Oris, K. Ryder, S. E. Belanger, T. Braunbeck, M. R. Embry, G. Whale, T. Norberg-King, A. Lillicrap, Regulatory aspects on the use of fish embryos in environmental toxicology, Integrated environmental assessment and management (6) (2010) 484-491.

[7] U. Strähle, S. Scholz, R. Geisler, P. Greiner, H. Hollert, S. Rastegar, A. Schumacher, I. Selderslaghs, C. Weiss, H. Witters, T. Braunbeck, Zebrafish embryos as an alternative to animal experiments - a commentary on the definition of the onset of protected life stages in animal welfare regulations, Reproductive Toxicology.

[8] J. Schindelin, I. Arganda-Carreras, E. Frise, V. Kaynig, M. Longair, T. Pietzsch, S. Preibisch, C. Rueden, S. Saalfeld, B. Schmid, J.-Y. Tinevez, D. J. White, V. Hartenstein, K. Eliceiri, P. Tomancak, A. Cardona, Fiji: an open-source platform for biological-image analysis, Nature Methods 9 (7) (2012) 676-682. doi:10.1038/nmeth.2019

URL http://www.nature.com/doifinder/10.1038/nmeth.2019

[9] B. Ruff, J. Marchant, A. Frost, Fish sizing and monitoring using a stereo image analysis system applied to fish farming, Aquacultural engineering 14 (2) (1995) 155-173.

540 [10] A. K. Morison, S. G. Robertson, D. C. Smith, An integrated system for production fish aging: image analysis and quality assurance, North American Journal of Fisheries Management 18 (3) (1998) 587-598. 
[11] N. Strachan, P. Nesvadba, A. R. Allen, Fish species recognition by shape analysis of images, Pattern Recognition 23 (5) (1990) 539-544.

[18] S. Xia, Y. Zhu, X. Xu, W. Xia, Computational techniques in zebrafish image processing and analysis, Journal of Neuroscience Methods 213 (1) (2013) 6-13. doi:10.1016/j.jneumeth.2012.11.009 
URL http://linkinghub.elsevier.com/retrieve/pii/ S0165027012004657

[19] C. Pylatiuk, D. Sanchez, R. Mikut, R. Alshut, M. Reischl, S. Hirth, W. Rottbauer, S. Just, Automatic zebrafish heartbeat detection and analysis for zebrafish embryos, Zebrafish 11 (4) (2014) 379-383.

[20] E. Jacob, M. Drexel, T. Schwerte, B. Pelster, Influence of hypoxia and of hypoxemia on the development of cardiac activity in zebrafish larvae, American journal of physiology. Regulatory, integrative and comparative physiology (283).

[21] R. P., Gills are needed for ionoregulation before they are needed for o2 uptake in developing zebrafish, danio rerio, The Journal of Experimental Biology (205).

[22] E. Puybareau, M. Léonard, H. Talbot, An automated assay for the evaluation of mortality in fish embryo, in: Mathematical Morphology and Its Applications to Signal and Image Processing, Vol. 9082 of Lecture Notes in Computer Science, Springer, Reykjavik, 2015, pp. 110-121.

[23] J. Serra, Image Analysis and Mathematical Morphology - Volume II : Theoretical Advances, Academic Press, London, 1988.

[24] P. Soille, Morphological Image Analysis, principles and applications, Springer, 1999.

[25] L. Najman, H. Talbot (Eds.), Mathematical Morphology: from theory to applications, ISTE-Wiley, London, UK, 2010, iSBN 978-1848212152.

[26] E. Puybareau, H. Talbot, M. Leonard, Automated heart rate estimation in fish embryo, in: Image Processing Theory, Tools and Applications (IPTA), International Conference on, Orleans, 2015, pp. 379-384.

[27] S. Van der Walt, J. L. Schönberger, J. Nunez-Iglesias, F. Boulogne, J. D. Warner, N. Yager, E. Gouillart, T. Yu, scikit-image: image processing in python, PeerJ 2 (2014) e453. 
[28] M. Couprie, L. Marak, H. Talbot, et al., The pink image processing library, in: European Python Scientific Conference, 2011.

URL http://www . pinkhq.com

[29] G. Bradski, et al., The opencv library, Doctor Dobbs Journal 25 (11) (2000) 120-126.

[30] H. Heijmans, Morphological image operators, Advances in Electronics and Electron Physics Series, Academic Press, Boston, 1994.

[31] J. Serra, Image analysis and mathematical morphology, Academic Press, 1982.

[32] L. Vincent, Morphological area openings and closings for grey-scale images, in: Shape in Picture, Springer, 1994, pp. 197-208.

[33] P. Soille, H. Talbot, Directional morphological filtering, IEEE Transactions on Pattern Analysis and Machine Intelligence 23 (11) (2001) 1313-1329.

[34] H. Blum, A transformation for extracting new descriptors of shape, in: W. Wathen-Dunn (Ed.), Models for the Perception of Speech and Visual Form, MIT Press, Cambridge, MA, 1967, pp. 362-380. URL http://pageperso.lif.univ-mrs.fr/ edouard.thiel/rech/ 1967-blum.pdf

[35] F. Meyer, S. Beucher, Morphological segmentation, Journal of Visual Communication and Image Representation 1 (1) (1990) 21-46.

[36] D. Marr, E. Hildreth, Theory of edge detection, Proceedings of the Royal Society of London B: Biological Sciences 207 (1167) (1980) 187-217.

[37] N. Otsu, A threshold selection method from gray-level histograms, Automatica 11 (285-296) (1975) 23-27.

[38] R. Deriche, Using canny's criteria to derive a recursively implemented optimal edge detector, International journal of computer vision 1 (2) (1987) $167-187$. 
[39] J. Cousty, G. Bertrand, L. Najman, M. Couprie, Watershed cuts: minimum spanning forests and the drop of water principle, IEEE transactions on Pattern Analysis and Machine Intelligence (2008) 1362-1374.

[40] R. A. Jarvis, On the identification of the convex hull of a finite set of points in the plane, Information Processing Letters 2 (1) (1973) 18-21.

[41] L. Vincent, Morphological grayscale reconstruction in image analysis: Applications and efficient algorithms, Image Processing, IEEE Transactions on 2 (2) (1993) 176-201.

[42] R. Kresch, D. Malah, Skeleton-based morphological coding of binary images, IEEE Transactions on Image Processing 7 (10) (1998) 1387-1399.

[43] D. G. Lowe, Object recognition from local scale-invariant features, in: IEEE International Conference on Computer Vision, Vol. 2, Kerkyra, Greece, 1999, pp. 1150-1157.

[44] C. Tomasi, R. Manduchi, Bilateral filtering for gray and color images, in: IEEE International Conference on Computer Vision, 1998, pp. 839-846.

[45] H. Talbot, H. Phelippeau, M. Akil, S. Bara, Efficient poisson denoising for photography, in: 16th IEEE International Conference on Image Process-

口 ing (ICIP), Cairo, Egypt, 2009, pp. 3881-3884. doi:10.1109/icip.2009. 5414042 